\title{
Construction of an adenoviral expression vector carrying FLAG and hrGFP-1 genes and its expression in bone marrow mesenchymal stem cells
}

\author{
G.X. Wang', L. Hü ${ }^{2}$, Z. Zhang ${ }^{3}$ and D.P. Liu $^{3}$ \\ ${ }^{1}$ Department of Pharmacology, Liaoning Medical University, Jinzhou, \\ Liaoning, China \\ ${ }^{2}$ Department of Orthopaedics, First People's Hospital of Jingmen, China \\ ${ }^{3}$ Department of Bone and Joint Surgery, \\ First Affiliated Hospital of Liaoning Medical University, Jinzhou, China \\ Corresponding author: D.P. Liu \\ E-mail: liudpcn@126.com
}

Genet. Mol. Res. 13 (1): 1070-1078 (2014)

Received January 18, 2013

Accepted July 12, 2013

Published February 20, 2014

DOI http://dx.doi.org/10.4238/2014.February.20.8

\begin{abstract}
The aim of this study was to construct an adenoviral expression vector for vascular endothelium growth factor 121 $\left(\mathrm{VEGF}_{121}\right)$-FLAG and humanized Renilla reniformis green fluorescent protein (hrGFP-1) genes, and to observe their expressions in bone marrow mesenchymal stem cells. Using pTG19T-VEGF ${ }_{121}$ as a template, polymerase chain reaction technology was adopted to mutate the VEGF $_{121}$ gene by removing the stop codon and inserting NotI and XhoI restriction sites both before and after the gene sequences. The resultant gene was then subcloned into a pMD19-T plasmid, the pMD19-TVEGF $_{121}$ and pShuttle-CMV-IRES-hrGFP-1 plasmids were doubledigested, and small and large fragments were linked after gel recovery to complete the construction of recombinant adenovirus vectors. After titer determination, the recombinant adenovirus vectors were used to affect
\end{abstract}


rabbit bone marrow mesenchymal stem cells, and fluorescence intensity was observed under fluorescence microscopy. Enzyme digestion identification and sequencing confirmed that the recombinant plasmids were successfully constructed, and observations under fluorescence microscopy showed significant expression of green fluorescent protein in recombinant adenovirus-infected bone marrow mesenchymal stem cells. The constructed adenoviral gene expression vectors carrying VEGF $_{121}$-FLAG and hrGFP-1 can be expressed in eukaryotic cells, which may be used for gene therapy of ischemic disorders.

Key words: Bone defect; Vascular endothelial growth factor 121; Adenovirus vector; Green fluorescent protein

\section{INTRODUCTION}

The formation and growth of blood vessels in the treatment of diseases of bone defects are important steps for the bone tissue regeneration process (Holstein et al., 2011; Levi et al., 2012; Nakagaki and Camilli, 2012). The factors promoting angiogenesis can be divided into direct-inducing and indirect-inducing factors, in which vascular endothelial growth factor (VEGF) is a strong direct vascular-inducing factor, and $\mathrm{VEGF}_{165}$ and $\mathrm{VEGF}_{121}$ play the most significant roles. VEGF ${ }_{165}$ is expressed as a half soluble and half insoluble protein in vivo, and can be secreted extracellularly, with part of the protein binding to the cell membrane or substrate. By contrast, $\mathrm{VEGF}_{121}$ is entirely soluble, does not bind to heparin weak acid, is completely secreted into the extracellular matrix, and is separated from the intercellular substance; thus, it plays a better paracrine role and promotes angiogenesis (He et al., 2010; Kinoshita et al., 2010).

VEGF is the most important growth factor promoting blood vessel growth in the body. It binds to vascular endothelial cell surface receptors through autocrine or paracrine activity to induce the establishment of new blood vessels (Simons et al., 2000; Li et al., 2009; Burger, 2010), supplies oxygen for bone defects, provides nutrients, and transports metabolic wastes, thus providing strong protection for local bone regeneration and metabolism. In addition, Flt1 receptors of VEGF are present on osteoblasts, and by acting on the strong expression Flt-1 receptor of osteoblasts, VEGF recruits chemokines, so that osteoblasts gather at the defect sites and differentiate into bone tissue under the action of VEGF (Mayr-Wohlfart et al., 2002; Singh et al., 2007; Kanczler et al., 2008; Kumar et al., 2010).

In the present study, to determine the specific angiogenesis and osteoblast bone differentiation promoting effects of VEGF, humanized Renilla reniformis green fluorescent protein (hrGFP-1) was used as a reporter gene in order to construct a recombinant adenovirus containing the VEGF $_{121}$-FLAG and hrGFP-1 gene expression system. The expression of bone marrow mesenchymal stem cells was then observed in vitro. Results of this study should provide an experimental basis for developing orthopedic gene therapy of ischemic disease.

\section{MATERIAL AND METHODS}

\section{Animals}

A clean-grade Japanese male rabbit weighing $1.2 \mathrm{~kg}$ was provided by the Experimental 
Animal Center, Liaoning Medical College (License No. SCXK, Liaoning, 2003-2007). This study was carried out in strict accordance with recommendations in the Guide for the Care and Use of Laboratory Animals of the National Institute of Health. The animal use protocol was reviewed and approved by the Institutional Animal Care and Use Committee (IACUC) of the First Affiliated Hospital of Liaoning Medical University.

\section{Cloning of VEGF}

Using pTG19T-VEGF ${ }_{121}$ as a template, polymerase chain reaction (PCR) technology was adopted to mutate the VEGF $_{121}$ gene by removing its stop codon TAG sequence and inserting NotI and XhoI restriction sites both before and after the gene sequences. Primer sequences were as follows: F: 5'-GCGGCCGCATGAACTTTCTGCTGTCTTG-3'; R: 5'-CTCGAGCCGCCTCGGCTTGTCACATT-3'. pTG19T-VEGF ${ }_{121}$ was used as a template to carry out PCR, and the reaction conditions were as follows: $98^{\circ} \mathrm{C}, 10 \mathrm{~s}, 30$ cycles; $55^{\circ} \mathrm{C}, 5$ s, 30 cycles; $72^{\circ} \mathrm{C}, 30 \mathrm{~s}, 30$ cycles; $72^{\circ} \mathrm{C}, 5 \mathrm{~min}, 1$ cycle, and products were visualized with agarose gel electrophoresis.

PCR products were recycled after gel cutting. PCR products were linked with the pMD19-T plasmid using Solution I from TaKaRa DNA Ligation Kit Ver.2.0. Competent cells was transformed, and plates were smeared and cultured at $37^{\circ} \mathrm{C}$ overnight. The plasmid was then extracted and sequenced.

\section{Plasmid construction and identification}

Double digestion with NotI and XhoI was conducted on plasmid pMD19-T- ${ }^{+} \mathrm{VEGF}_{121}$ and shuttle vector pShuttle-CMV-IRES-hrGFP-1, respectively. After recycling by cutting, they were mixed at a ratio of 10:1, T4 DNA ligase and buffer were then added, reacted at $16^{\circ} \mathrm{C}$ overnight, transformed into DH5 $\alpha$ bacteria, and plasmids were then extracted and cloned.

\section{Recombination of adenovirus and identification}

After PmeI digestion linearization, the adenovirus backbone plasmid pAdeasy-1 was transformed into BJ5183 bacteria to obtain BJ5183 bacteria containing pAdeasy-1, and the correctly identified shuttle vector pShuttle-CMV- ${ }^{+}$VEGF121-IRES-hrGFP-1 was transformed into the BJ5183 bacteria containing pAdeasy-1, and added to a cuvette after mixing well. Kanamycin-containing medium was then quickly added to the cuvette after electric shock, uniformly pipetted, and then inoculated into culture plates containing Kanamycin, and cultured overnight. Smaller colonies were picked out and amplified, the plasmid was extracted, and after the recombinant plasmid was digested with PacI, agarose gel electrophoresis was performed. The spectral shape was compared with that of the standard digestion spectral shape of the recombinant adenovirus to identify whether the obtained recombinant virus was correct.

\section{Packaging and amplification of the recombinant adenovirus}

To package the adenovirus, $293 \mathrm{~A}$ cells were cultured, and $4 \times 10^{5}$ cells were inoculated on 6-well plates. The recombinant adenovirus plasmid was digested with $\mathrm{PacI}$ and linearized, 
and was then purified by gel recycling. The linearized plasmid DNA was then transfected into 293 T cells using liposome Lipofectamine 2000. The medium was changed on day 4, cells were collected on day 11, and the virus was collected by repeated freezing and thawing. To amplify the adenovirus, $1 / 3$ of the obtained virus was used for infection of $293 \mathrm{~T}$ cells, and cells were collected after $48 \mathrm{~h}$. Amplification was repeated as such for a total of four times, all cells were collected, and were then frozen and thawed repeatedly to obtain the recombinant adenovirus.

\section{Determination of physical titer of recombinant viruses}

A large amount of the recombinant adenovirus was amplified and then gradiently centrifuged with $\mathrm{CsCl}_{2}$ twice, dialyzed to remove the $\mathrm{CsCl}_{2}$, and then stored in adenovirus storage buffer (10 mM Tris, $4 \%$ sucrose, $2 \mathrm{mM} \mathrm{MgCl}_{2}, \mathrm{pH} \mathrm{8.0)}$. Plaque analysis revealed that the titer of Ad- ${ }^{+} \mathrm{VEGF}_{121}$-IRES-hrGFP-1 was $6.50 \times 10^{9} \mathrm{pfu} / \mathrm{mL}$.

\section{Bone marrow extraction and identification}

A 2-month-old Japanese white rabbit was anaesthetized by intramuscular injection with $0.2 \mathrm{~mL} / \mathrm{kg}$ SU-MIAN-XIN II. The inner flank of the dual distal femur was selected as the puncture site, cropped, disinfected with iodophor, and then covered with a sterile towel. A total of $4.0 \mathrm{~mL}$ bone marrow was drawn with a No. 16 medullo-puncture needle by puncture. Under sterilized conditions, $4.0 \mathrm{~mL}$ bone marrow was injected into a $20-\mathrm{mL}$ sterile glass bottle, 4.0 $\mathrm{mL}$ Dulbecco's modified Eagle's medium (DMEM) containing 10\% fetal bovine serum was added, fully pipetted, and poured into eight $30-\mathrm{cm}^{2}$ culture flasks $(1.0 \mathrm{~mL}$ per flask) supplemented with $3.0 \mathrm{~mL}$ DMEM. Flasks were then placed into a constant temperature incubator at $37^{\circ} \mathrm{C}$ with $5 \% \mathrm{CO}_{2}$. The medium was changed after 5 days of culture, and then changed every 3 days thereafter. Cell growth was observed under an inverted microscope, and bone marrow mesenchymal stem cells, identified as visible cell spindle adherent cells, were subcultured 5 days later. When the third generation of cultured bone marrow mesenchymal stem cells was obtained, Ad- ${ }^{+} \mathrm{VEGF}_{121}$-IRES-hrGFP-1 was used to transfect the bone, and gene expression was observed under a fluorescence microscope.

\section{RESULTS}

\section{VEGF $_{121}$ clone}

Two bands were observed after the pMD19-T- ${ }^{+} \mathrm{VEGF}_{121}$ vector fragment was doubledigested with NotI and XhoI. One fragment, between 250-500 bp, was identified as the target gene (Figure 1A). Gene sequencing results showed that the stop codon of the $\mathrm{VEGF}_{121}$ gene fragment, GTA, was replaced by CTCGAG at the XhoI site after gene mutation, confirming that the stop codon was successfully removed (Figure 1B and C).

\section{Vector construction}

The shuttle vector, pShuttle-CMV-IRES-hrGFP-1, was identified after double-digestion with NotI and XhoI. Linear fragments were observed between 6557 and 9416 bp (Fig- 
ure 2A). At this point, as pShuttle-CMV-IRES-hrGFP-1 was an empty shuttle vector, there was still a fragment after double-digestion. For the linked shuttle vector pShuttle-CMV- of VEGF $_{121}$-IRES-hrGFP-1, agarose gel electrophoresis revealed the target band between 7500 and 10,000 bp on the gel (Figure 2B). Sequencing results showed that the target gene with stop codon removed was from site 71 to site 511, and was inserted into the shuttle vector in the correct reading frame. Bands were observed both at 3000 and 30,000 bp on the agarose gel after the recombinant adenovirus vector was digested with PacI, indicating that the shuttle vector had been successfully linked with the adenovirus genome, and that $\mathrm{pAd}^{+} \mathrm{VEGF}_{121}{ }^{-}$ IRES-hrGFP-1 had been successfully constructed (Figure 2C).

A

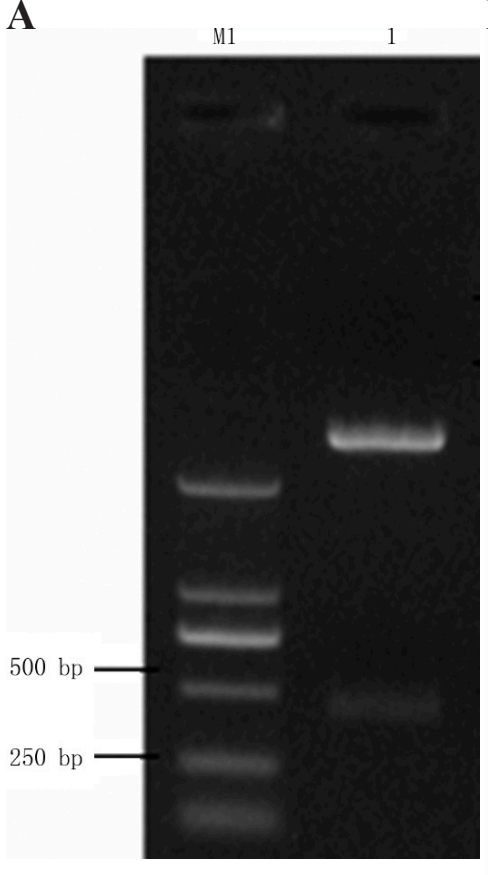

B

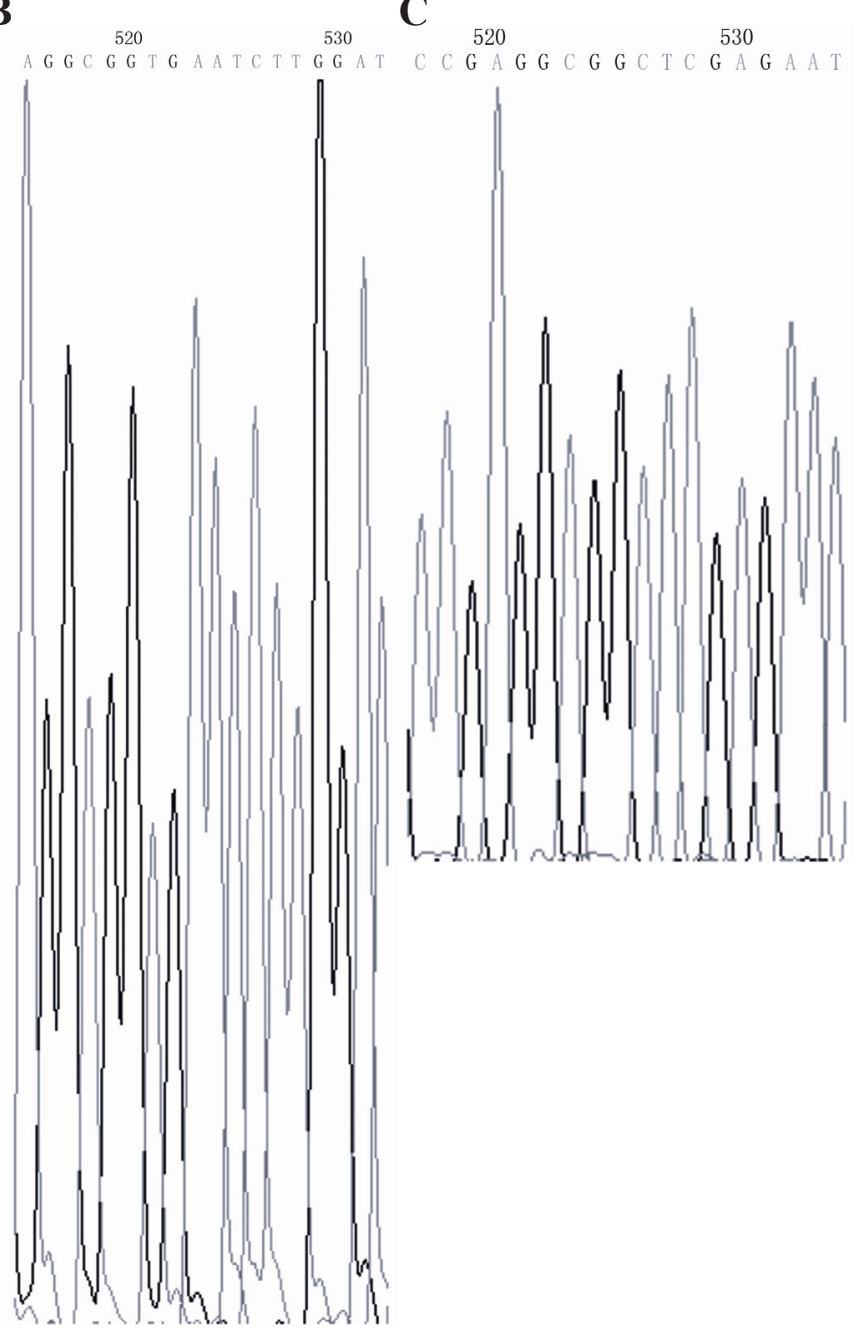

Figure 1. A. Result of pMD19-T- ${ }^{+} \mathrm{VEGF}_{121}$ digested by NotI and XhoI. Lane M1 = DL2000 DNA marker; lane $1=$ pMD19-T- ${ }^{+} V_{E G F}{ }_{121}$ NotI/XhoI. B. Partial sequencing map before mutation. C. Partial sequencing map after mutation. 
A

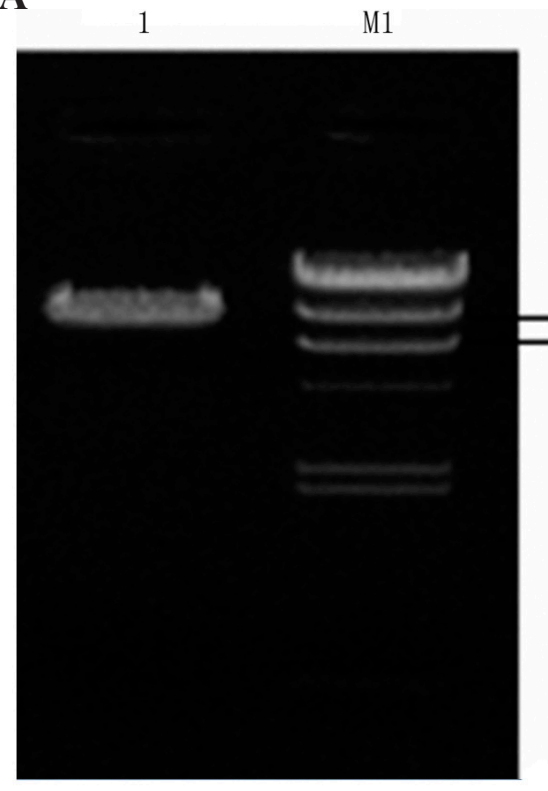

B

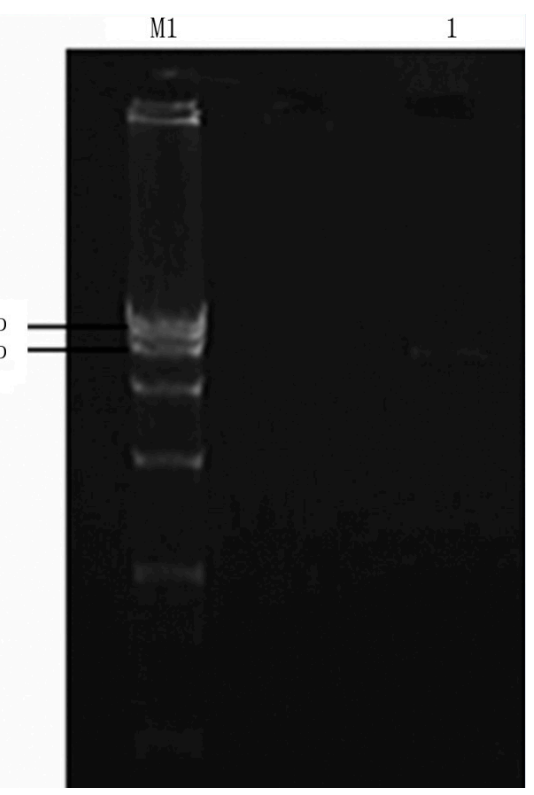

C

9416 bp $10000 \mathrm{bp}$
$6557 \mathrm{bp} 7500 \mathrm{bp}$

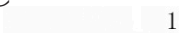

M1

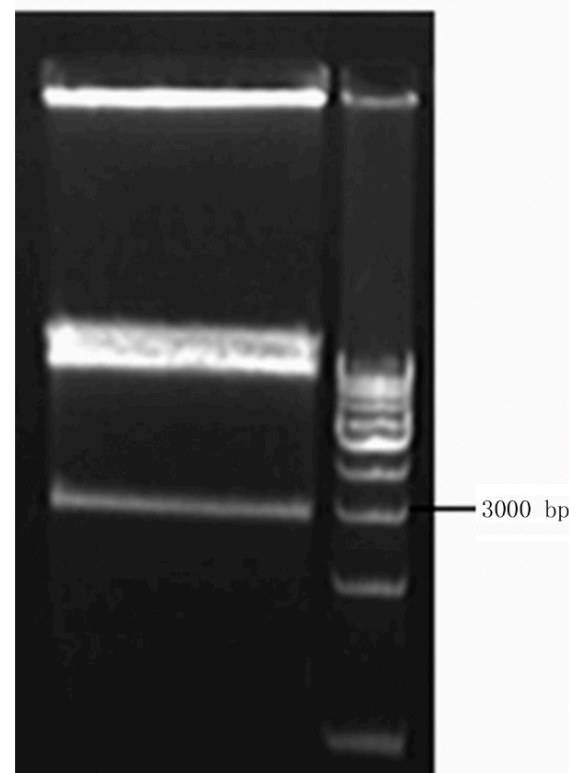

Figure 2. A. Electrophoresis result of pShuttle-CMV-IRES-hrGFP-1 after digestion by NotI and XhoI. Lane M1 $=\lambda$-HindIII digest DNA marker; lane $1=$ pShuttle-CMV-IRES-hrGFP-1 NotI/XhoI. B. Electrophoresis result of pShuttle CMV- ${ }^{+} V_{E G F}{ }_{121}$ IRES-hrGFP-1. Lane $M 1=$ DL15000 DNA marker; lane $1=$ pShuttle-CMV- ${ }^{+} V_{E G F}{ }_{121}{ }^{-}$ IRES-hrGFP-1. C. Electrophoresis result of recombinant pAd- ${ }^{+} \mathrm{EGF}_{121}$-hrGFP-1 cleaved by PacI. Lane M1 = 1 -kbp DNA Ladder marker; lane $1=3000$ - and 30000-bp DNA fragment. 


\section{Packaging of the recombinant adenovirus}

When Ad- ${ }^{+} V_{E G F}$-IRES-hrGFP-1 Lipofectamine 2000 was completely transfected into 293A cells for approximately 2 weeks, the vast majority of cells showed a cytopathic effect (CPE). The 293 cells were repeatedly infected, and on the third day after the 3rd infection, most of the 293T cells showed CPE, and fluorescence intensity was significantly enhanced (Figure 3).

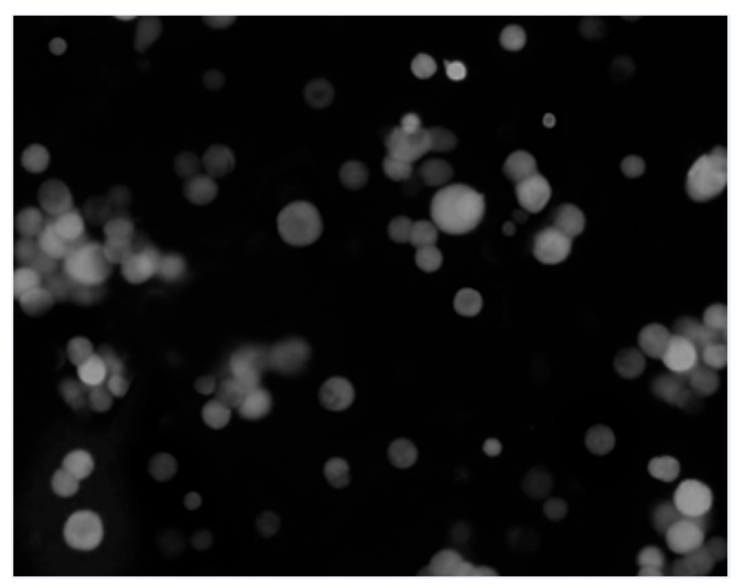

Figure 3. Fluorescence and cell changes effect after 2 weeks of post-transfection under fluorescence microscope.

\section{Gene expression}

When the third generation of bone marrow mesenchymal stem cells was transfected with Ad- ${ }^{+} V_{E G F}{ }_{121}$-IRES-hrGFP-1 for $48 \mathrm{~h}$, a strong green fluorescence effect was observed in the cells under a fluorescence microscope, indicating that the transfection effect was very good (Figure 4).

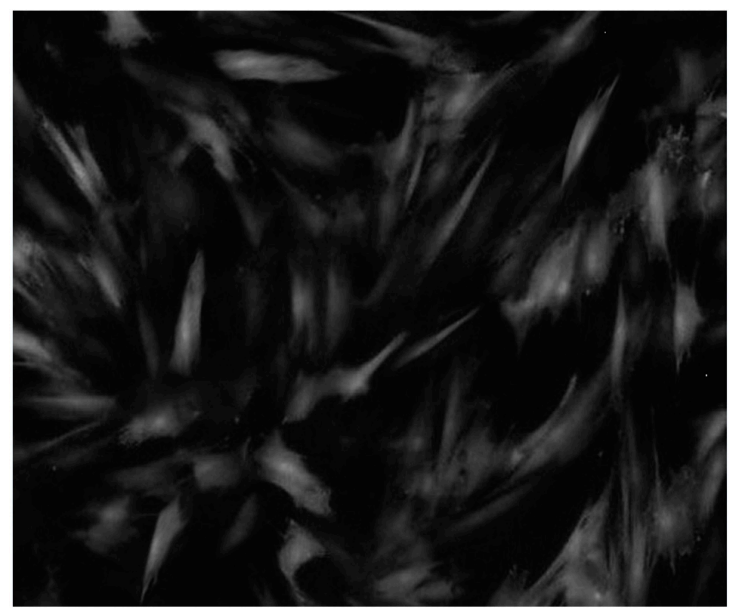

Figure 4. Fluorescence of bone marrow stromal stem cells $48 \mathrm{~h}$ after transfection. 


\section{DISCUSSION}

The green fluorescent protein gene is a reporter gene commonly used to study the intensity and duration of the in vivo expression of foreign genes during the process of gene therapy. A GFP variant, enhanced green fluorescent protein (EGFP), is most often used for reporter gene monitoring of in vivo gene expression and intracellular protein localization (Chen et al., 2009; Salunkhe et al., 2010; Kim et al., 2012). In the present study, we used the hrGFP-1, due to its advantages of being two times brighter than EGFP, more likely to be detected, and of smaller toxicity to mammalian cells.

After bone marrow mesenchymal stem cells were transfected with $\mathrm{Ad}-{ }^{+} \mathrm{VEGF}_{121}$, the IRES-hrGFP-1, the target gene, and hrGFP-1 were transcribed on the same mRNA. The internal ribosome entered the site of the IRES sequence and combined with the ribosomal body, and the translation of the target gene was simultaneously completed (Fernández-Miragall et al., 2009; Fitzgerald and Semler, 2009; Filbin and Kieft, 2009; Orrù et al., 2012). In this study, the stop codon of the $\mathrm{VEGF}_{121}$ gene was removed by mutation using the PCR technique, and then the NotI and XhoI digestion sites were added before and after the gene sequence, respectively. This allowed for the gene to be directionally linked at the location between NotI and XhoI in the polyclonal sites of the pShuttle, CMV-IRES-hrGFP-1. Before the internal ribosome of the shuttle vector entered the site of the IRES sequence, it contained a FLAG antigen label sequence. After the translation termination codon was removed, both the translation start codon of the $\mathrm{VEGF}_{121}$ gene sequence and the base sequence of FLAG epitope genes would not lead to a frameshift mutation of the FLAG antigen epitope gene, resulting in a read-through of the two genes, so that the fusion expression occurred for the two proteins, VEGF ${ }_{121}$ and FLAG.

As VEGF has six isomers, and there are no specific antibodies for $\mathrm{VEGF}_{12}$, the introduction of the FLAG epitope tag in this experiment was particularly important for the subsequent specificity testing of VEGF ${ }_{121}$. Since the application of recombinant adenovirus for human trials, there have been no reports of tumors or other genetic diseases caused by recombinant adenoviruses. Therefore, it can be used as a suitable vector of the VEGF gene and be introduced into host cells to meet the needs of VEGF during treatment for orthopedic ischemic disorders.

In the present study, we successfully constructed a recombinant adenovirus vector containing the VEGF $\mathrm{V}_{121}$ and hrGFP-1 genes, which could express the target genes in eukaryotic cells. This result was highly consistent with the expected experimental results of our research group, which has laid a foundation for in vivo topical applications of $\mathrm{VEGF}_{121}$ gene therapy for the treatment of bone ischemic disease.

As the successfully constructed adenovirus vector has not been transfected into the body for further in vivo experiments, the effect of the growth factor on blood vessels in orthopedic diseases remains unknown. Therefore, our future study will focus on such effects in further animal experiments.

\section{ACKNOWLEDGMENTS}

Research supported by the Key Talent Project of the Liaoning Provincial Health Bureau, China (\#2010921045). 


\section{REFERENCES}

Burger RA (2010). Role of vascular endothelial growth factor inhibitors in the treatment of gynecologic malignancies. $J$. Gynecol. Oncol. 21: 3-11.

Chen J, Sai SY, Vazin T, Coggiano M, et al. (2009). Human embryonic stem cells which express hrGFP in the undifferentiated state and during dopaminergic differentiation. Restor. Neurol. Neurosci. 27: 359-370.

Fernández-Miragall O, López de Quinto S and Martínez-Salas E (2009). Relevance of RNA structure for the activity of picornavirus IRES elements. Virus Res. 139: 172-182.

Filbin ME and Kieft JS (2009). Toward a structural understanding of IRES RNA function. Curr. Opin. Struct. Biol. 19: $267-276$.

Fitzgerald KD and Semler BL (2009). Bridging IRES elements in mRNAs to the eukaryotic translation apparatus. Biochim. Biophys. Acta 1789: 518-528.

He M, Cheng Y, Li W, Liu Q, et al. (2010). Vascular endothelial growth factor C promotes cervical cancer metastasis via up-regulation and activation of RhoA/ROCK-2/moesin cascade. BMC Cancer 10: 170.

Holstein JH, Orth M, Scheuer C, Tami A, et al. (2011). Erythropoietin stimulates bone formation, cell proliferation, and angiogenesis in a femoral segmental defect model in mice. Bone 49: 1037-1045.

Kanczler JM, Ginty PJ, Barry JJ, Clarke NM, et al. (2008). The effect of mesenchymal populations and vascular endothelial growth factor delivered from biodegradable polymer scaffolds on bone formation. Biomaterials 29: 1892-1900.

Kim YG, Park B, Ahn JO, Jung JK, et al. (2012). New cell line development for antibody-producing Chinese hamster ovary cells using split green fluorescent protein. BMC Biotechnol. 12: 24.

Kinoshita Y, Kasaoka S, Fujita M, Oshima C, et al. (2010). Synchronized changes in serum vascular endothelial growth factor during the clinical course of chronic systemic capillary leak syndrome. Intern. Med. 49: 791-794.

Kumar S, Wan C, Ramaswamy G, Clemens TL, et al. (2010). Mesenchymal stem cells expressing osteogenic and angiogenic factors synergistically enhance bone formation in a mouse model of segmental bone defect. Mol. Ther. 18: 1026-1034.

Levi B, Nelson ER, Hyun JS, Glotzbach JP, et al. (2012). Enhancement of human adipose-derived stromal cell angiogenesis through knockdown of a BMP-2 inhibitor. Plast. Reconstr. Surg. 129: 53-66.

Li R, Stewart DJ, von Schroeder HP, Mackinnon ES, et al. (2009). Effect of cell-based VEGF gene therapy on healing of a segmental bone defect. J. Orthop. Res. 27: 8-14.

Mayr-Wohlfart U, Waltenberger J, Hausser H, Kessler S, et al. (2002). Vascular endothelial growth factor stimulates chemotactic migration of primary human osteoblasts. Bone 30: 472-477.

Nakagaki WR and Camilli JA (2012). Bone tissue and muscle dystrophin deficiency in mdx mice. Joint Bone Spine 79: 129-133.

Orrù B, Cunniffe C, Ryan F and Schwartz S (2012). Development and validation of a novel reporter assay for human papillomavirus type 16 late gene expression. J. Virol. Methods 183: 106-116.

Salunkhe SS, Raiker VA, Rewanwar S, Kotwal P, et al. (2010). Enhanced fluorescent properties of an OmpT site deleted mutant of green fluorescent protein. Microb. Cell Fact. 9: 26.

Simons M, Bonow RO, Chronos NA, Cohen DJ, et al. (2000). Clinical trials in coronary angiogenesis: issues, problems, consensus: An expert panel summary. Circulation 102: E73-E86.

Singh AK, Gudehithlu KP, Patri S, Litbarg NO, et al. (2007). Impaired integration of endothelial progenitor cells in capillaries of diabetic wounds is reversible with vascular endothelial growth factor infusion. Transl. Res. 149: 282-291. 